\title{
In vitro and in vivo immunomodulating activities of Shoyu polysaccharides from soy sauce
}

\author{
HIROAKI MATSUSHITA $^{1}$, MAKIO KOBAYASHI ${ }^{1}$, RYO-ICHI TSUKIYAMA ${ }^{1}$ and KENJI YAMAMOTO $^{2}$ \\ ${ }^{1}$ Research Laboratory, Higashimaru Shoyu Co. Ltd., Tatsuno, Hyogo 679-4167; \\ ${ }^{2}$ Graduate School of Biostudies, Kyoto University, Sakyo-ku, Kyoto 606-8502, Japan
}

Received December 20, 2005; Accepted February 8, 2006

\begin{abstract}
We investigated the effects of Shoyu polysaccharides (SPS) prepared from soy sauce on immune functions in mice. SPS enhanced the consumption of glucose by peritoneal macrophages in vitro. In addition, oral administration of SPS to 6-week-old male BALB/c mice for 2 weeks at a dose of $3.0 \mathrm{mg} /$ day increased the capacity of peritoneal macrophages to consume glucose as compared to control mice. We observed the regulatory effect of SPS on the balance of Th1/Th2 cell responses in mice. In splenic lymphocytes stimulated with Con A in vitro, SPS significantly suppressed the production of IL-4 and enhanced that of IFN- $\gamma$. Furthermore, in mice treated with SPS at $3.0 \mathrm{mg}$ per day for 2 weeks, the balance of Th1/ Th2 cell responses was shifted to predominantly Th1 cell responses. These findings suggest that SPS effectively enhanced both macrophage and lymphocyte function in vitro and in vivo, and soy sauce would be a potentially promising food for enhancing host defenses.
\end{abstract}

\section{Introduction}

Soy sauce (Shoyu) is a traditional Japanese fermented seasoning available throughout the world (1). In Japanese Shoyu, soybeans and wheat are the two main raw materials, used in almost equal quantities. Proteins in the raw materials are completely degraded into peptides and amino acids by microbial proteolytic enzymes after fermentation and no allergens are present in the soy sauce (2). In contrast, polysaccharides originating from the cell wall of soybeans are resistant to enzymatic hydrolysis. These polysaccharides are present in soy sauce even after fermentation and are termed Shoyu polysaccharides (SPS) (3). Soy sauce contains approximately $1 \%(\mathrm{w} / \mathrm{v})$ SPS, and SPS exhibit potent anti-allergic activity in vitro and in vivo (3). Furthermore, oral supplementation of SPS was an effective

Correspondence to: Dr Makio Kobayashi, Research Laboratory, Higashimaru Shoyu Co. Ltd., 100-3 Tominaga, Tatsuno-cho, Tatsuno, Hyogo 679-4167, Japan

E-mail: mkobayashi@higashimaru.co.jp

Key words: macrophage, Th1/Th2 balance, lymphocyte, Shoyu polysaccharides (SPS), soy sauce intervention for patients with allergic rhinitis in two doubleblind placebo-controlled clinical studies $(4,5)$.

It has been shown that certain foods have a host defense function related to the immune system (6-9). In particular, several polysaccharides, such as dietary fibers (10), ß-glucan $(11,12)$, and marine algae $(13,14)$, have been shown to have immunomodulatory activities. These immunological functions are generally divided into two systems, natural and adaptive. In natural immunity, macrophages play diverse roles in the host defense mechanism against invasive insults. Their functions include the presentation of antigens to $\mathrm{T}$ and $\mathrm{B}$ cells, destruction of intracellular microbial pathogens, and generation and secretion of monokines (15). It is accepted that $\mathrm{CD}^{+} \mathrm{T}$ cell clones can be classified into two distinct populations, Th1 and Th2, based on patterns of cytokine production $(16,17)$. Th1 cells are mainly responsible for cellmediated immunity, while Th2 cells are responsible for humoral immunity. Each subset produces cytokines for self-amplification and cross-regulation. IFN- $\gamma$ produced by Th1 cells has two functions. Firstly, it activates macrophages, enhancing their ability to both phagocytize and destroy microbes. Secondly, it stimulates the production of $\mathrm{IgG}$ antibodies. The signature cytokines of Th2 cells are IL-4 and IL-5. IL-4 is the major inducer of IgE production in B cells. Studies have shown the importance of the balance of Th1/Th2 cells in disorders such as infectious and allergic diseases (17).

Therefore, in the present study, we examined the effect of SPS on the activation of macrophages as well as regulation of the balance of Th1/Th2 cell responses in order to clarify the immunomodulatory activities of SPS as a dietary functional component from soy sauce.

\section{Materials and methods}

Animals. Male BALB/c mice were obtained from Clea Japan, Inc. (Tokyo, Japan) and bred in our laboratories. The mice ranged in age from 8 to 10 weeks old when used. They were housed 5 or 10 to a cage in an animal room under a $12 \mathrm{~h}$ light/ dark cycle at a temperature of $24 \pm 1^{\circ} \mathrm{C}$ and $60 \pm 5 \%$ humidity and fed a CE-2 basal diet (Clea Japan, Inc.) and water ad libitum. After a week of acclimation, they were used for the experiment.

Preparation of Shoyu polysaccharides (SPS). Soy sauce (Shoyu) was fermented by Higashimaru Shoyu Co., Ltd. (Tatsuno, Hyogo, Japan) as described previously $(1,2)$. Shoyu 
polysaccharides (SPS) were prepared according to the method of Kikuchi and Sugimoto (18). SPS were prepared for the dialysate from soy sauce as follows (2): $10 \mathrm{ml}$ of soy sauce in seamless cellulose tubing (small size $18,25 \AA$ pore size, 12,000 molecular weight cut-off; Wako Pure Chemical Industries Ltd., Osaka, Japan) was dialyzed overnight in water at $4^{\circ} \mathrm{C}$ and then freeze-dried.

Isolation of macrophages. Cell suspensions of peritoneal macrophages were prepared as described by Tanaka et al (6). Eight-week-old male mice were injected intraperitoneally with $400 \mu \mathrm{l}$ of autoclaved $1 \%$ glycogen from bovine liver (Nacalai Tesque Inc., Kyoto, Japan) and sacrificed 3 days later. Macrophages were isolated from the peritoneal cavity by lavage with $7 \mathrm{ml}$ of ice-cold Hanks' Solution (HBSS; Gibco BRL, Life Technologies, Paisley, Scotland). After centrifugation at $300 \mathrm{x} \mathrm{g}$ for $10 \mathrm{~min}$ at $4^{\circ} \mathrm{C}$, the cell pellet was washed twice with $7 \mathrm{ml}$ of ice-cold HBSS and then resuspended in $1.5 \mathrm{ml}$ of RPMI-1640 medium (Gibco BRL) containing $10 \%$ heat-inactivated fetal bovine serum (FBS; Gibco BRL), $100 \mathrm{U} / \mathrm{ml}$ of penicillin (Sigma Chemical Co., St. Louis, MO, USA) and $100 \mu \mathrm{g} / \mathrm{ml}$ of streptomycin (Sigma Chemical Co.). This medium was termed RPMI-10\% FBS. The viability of peritoneal macrophages as determined with the trypan blue dye exclusion test was $>98 \%$. The cells were cultured at $4 \times 10^{5}$ cells $/ 200 \mu 1$ in 96 -well culture plates (Nalge Nunc International, Roskilde, Denmark) for $2 \mathrm{~h}$ until adhering to the plates. The obtained macrophage monolayers were washed 3 times with warm HBSS at $37^{\circ} \mathrm{C}$ and added to $100 \mu 1$ of RPMI-10\% FBS. Next, $100 \mu 1$ of the test sample dissolved in RPMI-10\% FBS was added to the cell suspension and the mixture was incubated for $72 \mathrm{~h}$ in a humidified $5 \%$ $\mathrm{CO}_{2}$ incubator at $37^{\circ} \mathrm{C}$. The culture supernatant was harvested to determine glucose consumption. Lipopolysaccharide (LPS from Salmonella typhimurium IL7261; Sigma Chemical Co.) was used as a macrophage-activator $(13,14)$.

Assay of glucose consumption in peritoneal macrophages. The amount of glucose remaining in the supernatant of the peritoneal macrophages was determined using a commercial glucose B-test kit (439-90901; Wako Pure Chemical Industries Ltd.).

Preparation of splenic lymphocytes. The procedure used to collect the spleen cells was a modification of that described by Yang et al (19). Briefly, organs were aseptically removed from the mice and placed in $5 \mathrm{ml}$ of RPMI-1640 medium in a plastic dish. After being gently dispersed using a 5-ml sterile syringe plunger, the cell suspensions were passed through a 200-mesh nylon sieve. Erythrocytes from the spleen cell suspensions were lysed with $0.83 \% \mathrm{NH}_{4} \mathrm{Cl}$. After centrifugation at $600 \mathrm{x} \mathrm{g}$ for $10 \mathrm{~min}$, the cell pellet was washed with $6 \mathrm{ml}$ of HBSS and resuspended in $2 \mathrm{ml}$ of RPMI-10\% FBS. The cell suspensions were passed through a 200-mesh nylon sieve, and adjusted to $1 \times 10^{7}$ viable cells per $\mathrm{ml}$. The viability of splenic lymphocytes as determined with the trypan blue dye exclusion test was $>98 \%$.

Proliferation assay. The spleen cells were plated at $1 \times 10^{6}$ cells/ $50 \mu 1$ in $96-w e l l$ culture plates and then $50 \mu 1$ of the test

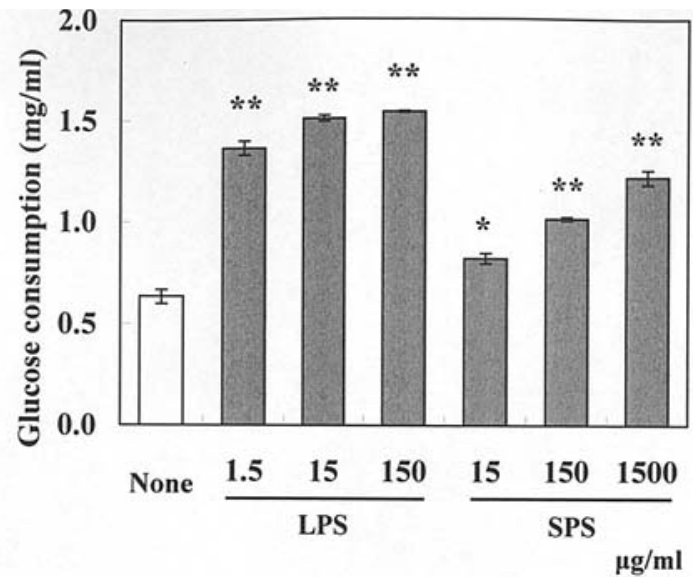

Figure 1. In vitro macrophage-stimulating activity of SPS. For details of the sample preparation and assay of glucose consumption by peritoneal macrophages, see Materials and methods. LPS from the microbial cell wall, which is known to activate macrophages, was used as a positive control in this experiment. Peritoneal macrophages, obtained from mice 3 days after an intraperitoneal injection of glycogen, were cultured for $72 \mathrm{~h}$ at $37^{\circ} \mathrm{C}$. The glucose remaining in the culture supernatant was assayed using a glucose Btest (Wako). Each value is the average of triplicate cultures, and each bar indicates the mean $\pm \mathrm{SE}(\mathrm{n}=5)$. Significantly different from the control (none) at ${ }^{*} \mathrm{P}<0.05$ or ${ }^{* *} \mathrm{P}<0.01$ (Student's t-test).

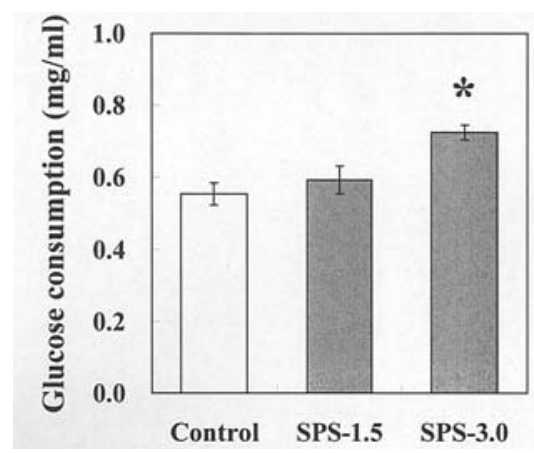

Figure 2. In vivo effect of SPS on glucose consumption in peritoneal macrophages stimulated by LPS. For details of the sample preparation and assay of glucose consumption, see Materials and methods. Mice were fed ad libitum with a CE-2 basal diet containing 0.05 or $0.10 \%(\mathrm{w} / \mathrm{w})$ SPS for 2 weeks. It was estimated that the oral dose of SPS was approximately $1.5 \mathrm{mg}$ (SPS-1.5) or 3.0 (SPS-3.0) mg per day per mouse. The control group was fed the basal diet only. Mice were sacrificed on day 14, and peritoneal macrophages from control and SPS-treated mice were incubated with $1 \mu \mathrm{g} / \mathrm{ml}$ of LPS for $72 \mathrm{~h}$ at $37^{\circ} \mathrm{C}$. The glucose in culture supernatants was measured with the glucose B-test (Wako). Each value is the average of triplicate cultures, and each bar indicates the mean $\pm \operatorname{SE}(n=5)$. Significantly different from the control at ${ }^{*} \mathrm{P}<0.05$ (Student's t-test).

sample dissolved in RPMI-1640 medium was added to the cell suspension. The plates were placed in a humidified $5 \%$ $\mathrm{CO}_{2}$ incubator at $37^{\circ} \mathrm{C}$ for $48 \mathrm{~h}$, and $10 \mu \mathrm{l}$ of 5-bromo-2'deoxyuridine (10 mM; Roche Diagnostics GmbH, Mannheim, Germany) in phosphate-buffered saline (PBS; pH 7.4) was added to each well $6 \mathrm{~h}$ before the end of the incubation. After incubation, the proliferation of cells was assayed using cell proliferation ELISA, BrdU (1647229; Roche Diagnostics $\mathrm{GmbH})$. A stimulation index (S.I.) was calculated according to the following equation: S.I. $=($ mean OD at $570 \mathrm{~nm}$ of the cells with a test sample)/(mean OD at $570 \mathrm{~nm}$ of the cells without a test sample). 
A

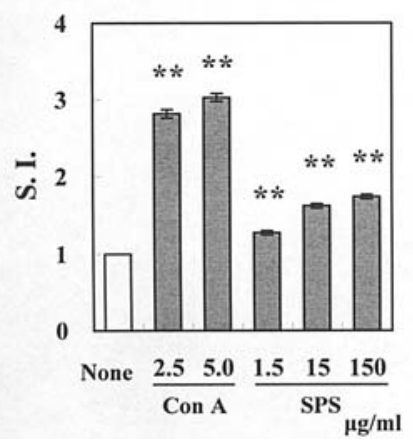

B

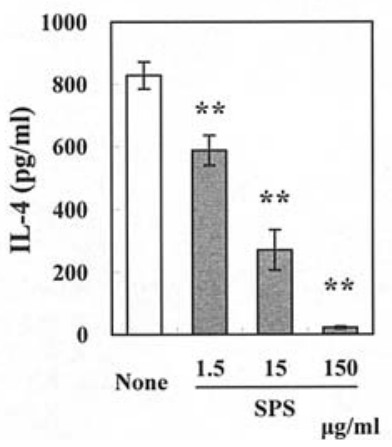

C

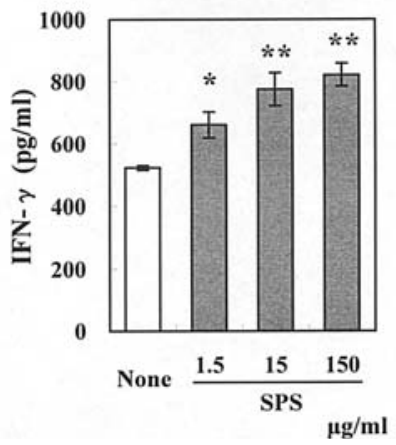

D

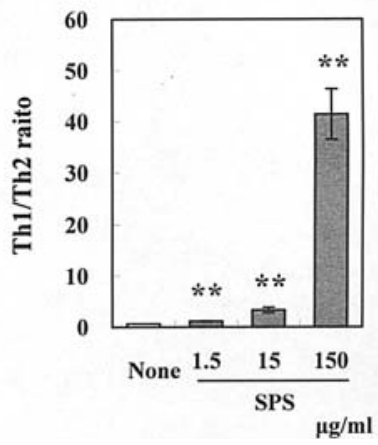

Figure 3. In vitro effect of SPS on proliferative responses (A), cytokine production (B and C), and the balance of Th1/Th2 cell responses (D) in splenic lymphocytes. For details of the sample preparation and assay of the proliferation and cytokine production of splenic lymphocytes, see Materials and methods. (A) Con A, a lymphocyte mitogen, was used as a positive control in this experiment. The spleen cells were incubated with the test sample in a humidified 5\% $\mathrm{CO}_{2}$ incubator at $37^{\circ} \mathrm{C}$ for $48 \mathrm{~h}$. The proliferation of cells was then assayed using cell proliferation ELISA, BrdU. A stimulation index (S.I.) was calculated using the following equation: S.I. = (mean OD at $570 \mathrm{~nm}$ of the cells with a test sample)/(mean OD at $570 \mathrm{~nm}$ of the cells without a test sample). (B and C) The spleen cells were incubated with the test sample in the presence of $5 \mu \mathrm{g} / \mathrm{ml} \mathrm{Con} \mathrm{A}$ in a humidified $5 \% \mathrm{CO}_{2}$ incubator at $37^{\circ} \mathrm{C}$ for $48 \mathrm{~h}$. The IFN- $\gamma$ and IL- 4 concentrations in the culture supernatant were determined using the ELISA system. (D) Th1/Th2 = IFN- $\gamma / \mathrm{IL}-4$. Each value is the average of triplicate cultures, and each bar indicates the mean $\pm \mathrm{SE}(\mathrm{n}=5)$. Significantly different from the control (none) at ${ }^{*} \mathrm{P}<0.05$ or ${ }^{* *} \mathrm{P}<0.01(\mathrm{Student}$ 's $\mathrm{t}$-test).

Table I. Effects of SPS on body weight of mice.

\begin{tabular}{lccc}
\hline & & \multicolumn{2}{c}{ Body weight $(\mathrm{g})$} \\
\cline { 3 - 4 } Group & $\mathrm{N}$ & Initial & After 14 days \\
\hline Control & 5 & $25.01 \pm 0.26$ & $27.24 \pm 0.30$ \\
SPS 1.5 & 5 & $25.26 \pm 0.46$ & $27.59 \pm 0.62$ \\
SPS 3.0 & 5 & $25.02 \pm 0.21$ & $27.89 \pm 0.23$
\end{tabular}

Each value represents the mean \pm SE. N, number of mice used. No significant differences in body weight were observed between groups.

Production of interferon- $\gamma(I F N-\gamma)$ and interleukin-4 (IL-4). The spleen cells were plated at $1 \times 10^{6}$ cells $/ 100 \mu 1$ in 96 -well culture plates, and $100 \mu 1$ of the test sample dissolved in RPMI-1640 medium containing $10 \mu \mathrm{g} / \mathrm{ml}$ of Concanavalin A (Con A; Wako Pure Chemical Industries Ltd.) was added to the cell suspension. After incubation in a humidified $5 \% \mathrm{CO}_{2}$ incubator at $37^{\circ} \mathrm{C}$ for $48 \mathrm{~h}$, the IFN- $\gamma$ and IL- 4 concentrations in the culture supernatant were determined using the ELISA system (mouse IFN- $\gamma$ and mouse IL-4; Bio Source International Inc., Camarillo, CA).

In vivo experiments. A total of 15 male 6-week-old BALB/c mice (weighing 20-23 g) were used for this experiment. After preliminary breeding for 4 days, the mice were divided into 3 groups of 5 . Mice in the test group were fed ad libitum with a CE-2 basal diet containing 0.05 or $0.10 \%$ (w/w) SPS for 2 weeks. It was estimated that the oral dose of SPS was approximately $1.5 \mathrm{mg}$ (SPS-1.5) or 3.0 (SPS-3.0) mg per day per mouse. The control group was fed the basal diet only. Mice were sacrificed on day 14 , and peritoneal macrophages were isolated from the peritoneal cavity as described above. The cells were cultured at $4 \times 10^{5}$ cells $/ 200 \mu 1$ in $96-w e l l$ culture plates for $2 \mathrm{~h}$ until adhering to the plates and washed 3 times with warm HBSS at $37^{\circ} \mathrm{C}$. The obtained macrophage monolayers were cultured for $72 \mathrm{~h}$ in a humidified $5 \% \mathrm{CO}_{2}$ incubator at $37^{\circ} \mathrm{C}$ in $200 \mu \mathrm{l}$ of RPMI-10\% FBS containing $1 \mu \mathrm{g} / \mathrm{ml}$ of LPS. The culture supernatant was then harvested to determine glucose consumption. Next, splenocytes were prepared from mice as outlined above. The spleen cells were plated at $5 \times 10^{5}$ cells $/ 100 \mu 1$ in 96 -well culture plates in the presence or absence of $5 \mu \mathrm{g} / \mathrm{ml}$ of Con A. The plates were placed in a humidified $5 \% \mathrm{CO}_{2}$ incubator at $37^{\circ} \mathrm{C}$ for $48 \mathrm{~h}$, and $10 \mu 1$ of 5-bromo-2'-deoxyuridine (10 mM) in PBS (pH 7.4) was added to each well $6 \mathrm{~h}$ before the end of the incubation. After incubation, the proliferation of cells was assayed using cell proliferation ELISA, BrdU. A stimulation index (S.I.) was calculated according to the following equation: S.I. = (mean OD at $570 \mathrm{~nm}$ of the cells stimulated with Con A)/ (mean OD at $570 \mathrm{~nm}$ of the cells not stimulated with Con A). In addition, the spleen cells $\left(5 \times 10^{5}\right.$ cells $\left./ 200 \mu \mathrm{l}\right)$ were incubated in the presence of $5 \mu \mathrm{g} / \mathrm{ml}$ of Con A in a humidified $5 \% \mathrm{CO}_{2}$ incubator at $37^{\circ} \mathrm{C}$ for $48 \mathrm{~h}$. After incubation, the IFN- $\gamma$ and IL-4 concentrations in the culture supernatant were determined using the ELISA system.

\section{Results}

Activation of macrophages by SPS. Fig. 1 shows the effect of SPS on the capacity of the peritoneal macrophage to consume glucose in vitro. LPS from the microbial cell wall, which is known to activate macrophages, was used as a positive control in this experiment. As shown in Fig. 1, SPS and LPS enhanced glucose consumption in macrophages compared with the sample-free culture (none). Next, the effect of SPS was examined in vivo. It was estimated that the oral dose of SPS was approximately 1.5 or $3.0 \mathrm{mg}$ per day, as the mice ingested approximately $3 \mathrm{~g}$ of the diet per day throughout the feeding period. Table I shows the effect of SPS on the body weight of the mice. There were no significant differences in body weight between the groups. The capacity of the peritoneal macrophages to consume glucose in the mice fed SPS is indicated in Fig. 2. The capacity of the macrophages from the 
A

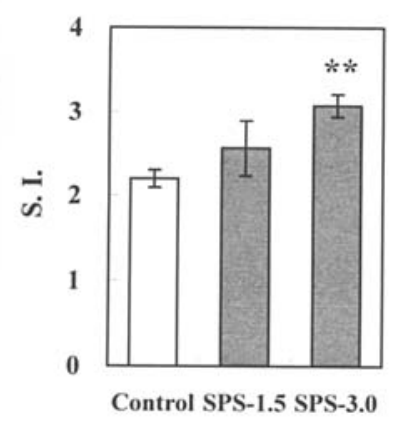

B

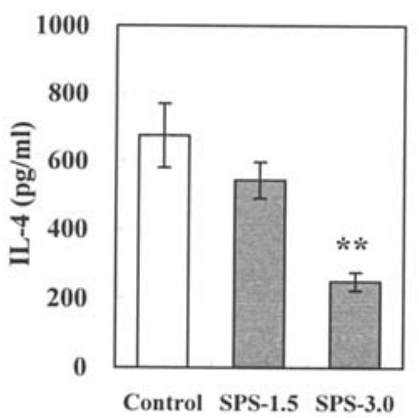

C

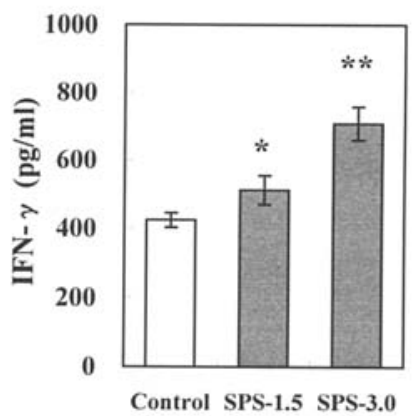

D

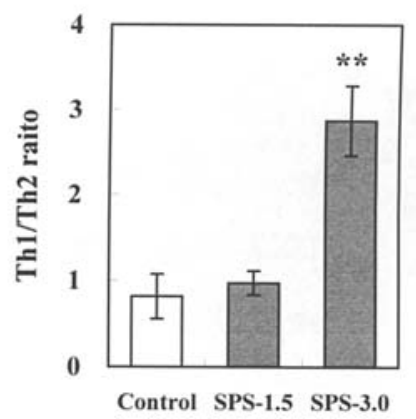

Figure 4. In vivo effect of SPS on proliferative responses (A), cytokine production (B and C), and the balance of Th1/Th2 cell responses (D) in splenic lymphocytes. For details of the sample preparation and assay of the proliferation and cytokine production of splenic lymphocytes, see Materials and methods. Mice were fed ad libitum with either a basal diet containing SPS or a basal diet only for 2 weeks. Mice were sacrificed on day 14 , and splenocytes were prepared. (A) The spleen cells were stimulated by the addition of $5 \mu \mathrm{g} / \mathrm{ml}$ of Con A in a humidified $5 \% \mathrm{CO}_{2}$ incubator at $37^{\circ} \mathrm{C}$ for $48 \mathrm{~h}$. The proliferation of cells was then assayed using cell proliferation ELISA, BrdU. A stimulation index (S.I.) was calculated using the following equation: S.I. $=($ mean OD at $570 \mathrm{~nm}$ of the cells stimulated with Con A)/(mean OD at $570 \mathrm{~nm}$ of the cells not stimulated with Con A). (B and C) The spleen cells were incubated in the presence of $5 \mu \mathrm{g} / \mathrm{ml}$ of Con $\mathrm{A}$ in a humidified $5 \% \mathrm{CO}_{2}$ incubator at $37^{\circ} \mathrm{C}$ for $48 \mathrm{~h}$. The IFN- $\gamma$ and IL- 4 concentrations in the culture supernatant were then determined using the ELISA system. (D) Th1/Th2 = IFN- $\gamma / \mathrm{IL}-4$. Each value is the average of triplicate cultures, and each bar indicates the mean \pm SE ( $\mathrm{n}=5$ ). Significantly different from the control (none) at ${ }^{*} \mathrm{P}<0.05$ or ${ }^{* *} \mathrm{P}<0.01$ (Student's t-test).

SPS-3.0 group was significantly $(\mathrm{P}<0.05)$ higher than that of the macrophages from the control group.

Regulatory effect of SPS on the balance of Th1/Th2 cell responses in vitro. The stimulation index values are indicated in Fig. 3A. Con A, a lymphocyte mitogen $(6,20)$, was used as a positive control in this experiment. The proliferation of splenic lymphocytes in the presence of SPS, as well as Con $\mathrm{A}$, increased significantly $(\mathrm{P}<0.01)$ according to concentration. The effect of SPS on the production of IFN- $\gamma$ and IL-4 in splenic lymphocytes stimulated with Con A is shown in Fig. 3B and C. When the lymphocytes were cultured without Con A, IFN- $\gamma$ and IL-4 were not detected in the supernatant (data not shown). Therefore, cells were cultured in medium supplemented with Con A. SPS significantly $(\mathrm{P}<0.05)$ reduced the production of $\mathrm{IL}-4$ in splenic lymphocytes, and its inhibitory effect was concentrationdependent. In contrast, IFN- $\gamma$ production was significantly $(\mathrm{P}<0.05-0.01)$ increased by SPS, and again the effect was concentration-dependent. IFN- $\gamma$ and IL-4 were used as markers for the generation of Th1 cells and Th2 cells. The results on the balance of $\mathrm{Th} 1 / \mathrm{Th} 2$ cell responses of splenic lymphocytes stimulated by Con A are shown in Fig. 3D. The responses were shifted to predominantly the Th1 cell type in the presence of SPS.

Regulatory effect of SPS on the balance of Th1/Th2 cell responses in vivo. Fig. 4A shows the stimulation index values in the three groups. The proliferation of splenic lymphocytes from mice fed SPS (SPS-3.0) was significantly $(\mathrm{P}<0.01)$ greater than that of mice fed the basal diet only (control). The levels of IFN- $\gamma$ and IL-4 produced by splenic lymphocytes stimulated with Con A are shown in Fig. 4B and C. Compared with the control group, the SPS-3.0 group exhibited a significant $(\mathrm{P}<0.05)$ decrease in the production of IL-4 by splenic lymphocytes stimulated with Con A. On the other hand, the production of IFN- $\gamma$ by splenic lymphocytes stimulated with Con A was significantly $(\mathrm{P}<0.05)$ greater in the SPS-treated group than the control group. The results on the balance of Th1/Th2 cell responses of splenic lymphocytes stimulated by Con A are shown in Fig. 4D. In the SPS-treated group, there was a shift to predominantly Th1 cell responses compared to the control group.

\section{Discussion}

Macrophages play an important role in the host defense system. In this study, SPS enhanced glucose consumption in peritoneal macrophages treated with glycogen in vitro. In addition, oral administration of SPS to mice increased the capacity of peritoneal macrophages to consume glucose in vivo. These results suggest that SPS activate peritoneal macrophages. It is accepted that glucose consumption increases as a result of the activation of macrophages at the priming stage (15). Since glucose is used to produce ATP and NADPH for the expression of active oxygen species and phagocytosis $(11,20)$, it is likely that SPS enhance the pentose phosphate pathway in peritoneal macrophages. Belardelli reported that all cytokines produced by Th1 cells have some positive role in the activation of macrophages, whereas cytokines such as IL-4 by Th2 cells exhibit suppressive effects on macrophage functions (21). Thus, the balance of Th1 and Th2 cell responses is cross-linked with macrophage function and SPS might directly enhance macrophages and/or cause a shift to predominantly Th1 cell responses in mice.

In 1972, Kikuchi and Yokotsuka purified polysaccharides from soy sauce and investigated their properties in detail (22). The cell wall polysaccharides of the soybean, one of the main raw materials of soy sauce, contain a large amount of galacturonic acid $(18,22)$. Some 30 years later, it was reported that polysaccharides, such as pectic substances, are present in soy sauce $(18,22)$ and that SPS have anti-allergic activities. The inhibitory effects of SPS on hyaluronidase, which is known to be related to inflammation and allergic responses, 
were as potent as those of an anti-allergic medicine, disodium cromoglycate (3). SPS also significantly inhibited the release of histamine from rat basophilic leukemia (RBL-2H3) cells (3) which had been induced by an antigen. In addition, orally administered SPS had a significant suppressive effect on passive cutaneous anaphylaxis induced in the ears of mice (3). All of these findings make it clear that SPS suppress the release of histamine in the presence of $\mathrm{IgE}$.

It was reported that the $\mathrm{Th} 1 / \mathrm{Th} 2$ cell response was shifted to a predominantly Th2 cell response in allergic diseases, and production of $\operatorname{IgE}$ by $\mathrm{B}$ cells was thus increased in these diseases (17). Lim et al reported that pectin could alleviate type I allergic reactions by enhancing lymphocyte function (10). Shida et al and Fujiwara et al showed that certain lactic acid bacteria inhibited the rise in serum IgE levels by improving the Th1/Th2 balance $(23,24)$. In the present study, we examined the regulatory effect of SPS on the balance of Th1/Th2 cell responses in vitro and in vivo. In splenic lymphocytes stimulated by Con A, SPS significantly suppressed the production of IL-4 and enhanced that of IFN- $\gamma$. Thus, in mice treated with SPS, there was a shift toward predominantly Th1 cell responses. Therefore, SPS may play a role in the suppression of IgE production by B cells through adaptation of the balance of Th1/Th2. In short, SPS exert part of their anti-allergic effect through the regulation of immune function. In conclusion, we have shown that SPS effectively enhanced both macrophage and lymphocyte function, and soy sauce would be a potentially promising food for enhancing host defenses.

\section{Acknowledgements}

The authors are grateful to Dr Seiji Kawamoto (Hiroshima University) and Dr Hiroaki Matsushita (Osaka University) for technical suggestions and helpful discussions. The authors are also grateful to Dr Kohei Ushio (Research Laboratory, Higashimaru Shoyu Co. Ltd.) for the critical reading of this manuscript and helpful advice. This work was supported in part by a subsidy from the Ministry of Agriculture, Forestry and Fisheries of Japan (Technical Development Program for making agribusiness in the form of utilizing the concentrated know-how from the private sector).

\section{References}

1. Yokotsuka T: Soy sauce biochemistry. Adv Food Res 30: 195-329, 1986.

2. Kobayashi M, Hashimoto Y, Taniuchi S and Tanabe S: Degradation of wheat allergen in Japanese soy sauce. Int J Mol Med 13: 821-827, 2004.

3. Kobayashi M, Matsushita H, Yoshida K, Tsukiyama R, Sugimura $\mathrm{T}$ and Yamamoto $\mathrm{K}$ : In vitro and in vivo anti-allergic activity of soy sauce. Int J Mol Med 14: 879-884, 2004.

4. Kobayashi M, Matsushita H, Shioya I, Nagai M, Tsukiyama R, Saito M, Sugita T, Sugimura T and Yamamoto K: Quality of life improvement with soy sauce ingredients, shoyu polysaccharides, in perennial allergic rhinitis: a double-blind placebocontrolled clinical study. Int J Mol Med 14: 885-889, 2004.

5. Kobayashi M, Matsushita H, Tsukiyama R, Saito M and Sugita T: Shoyu polysaccharides from soy sauce improve quality of life for patients with seasonal allergic rhinitis: a double-blind placebocontrolled clinical study. Int J Mol Med 15: 463-467, 2005.
6. Tanaka T, Sugiura H, Inaba R, Nishikawa A, Murakami A, Koshimizu K and Ohigashi H: Immunomodulatory action of citrus auraptene on macrophage functions and cytokine production of lymphocytes in female BALB/c mice. Carcinogenesis 20: 1471-1476, 1999.

7. Oka H, Emori Y, Kobayashi N, Hayashi Y and Nomoto K: Suppression of allergic reactions by royal jelly in association with the restoration of macrophage function and the improvement of Th1/Th2 cell responses. Int Immunopharmacology 1: 521-532, 2001.

8. Hosono A, Otani H, Yasui $\mathrm{H}$ and Watanuki M: Impact of fermented milk on human health: Cholesterol-lowering and immunomodulatory properties of fermented milk. Animal Sci J 73: 241-256, 2002.

9. Ike K, Uchida Y, Nakamura T and Imai S: Induction of interferon-gamma (IFN- $\gamma$ ) and T helper 1 (Th1) immune response by bitter gourd extract. J Vet Med Sci 67: 521-524, 2005.

10. Lim BO, Yamada K, Nonaka M, Kuramoto Y, Hung P and Sugano M: Dietary fibers modulate indices of intestinal immune function in rats. J Nutr 127: 663-667, 1997.

11. Kiho T, Matsushita M, Usui S and Ukai S: Biological activities of (1-3)-B-D-Glucans with reducing glucose side chains. Biosci Biotechnol Biochem 62: 570-572, 1998.

12. Kawaguchi M, Shiomi R, Tabata K, Kosuge Y, Mizumachi K and Kurisaki J: Enhancement of Th1 immune response in mice by the ingestion of Suehirotake (Schizophyllum commune Fries) mycelium. Nippon Shokuhin Kagaku Kogaku Kaishi 51: 102-105, 2004.

13. Yoshizawa Y, Enomoto A, Todoh H, Ametani A and Kaminogawa S: Activation of murine macrophages by polysaccharide fractions from marine algae (Porphyra yezoensis). Biosci Biotechnol Biochem 57: 1862-1866, 1993.

14. Yoshizawa Y, Ametani A, Tsunehiro J, Nomura K, Itoh M, Fukui $\mathrm{F}$ and Kaminogawa S: Macrophage stimulation activity of the polysaccharide fraction from a marine alga (Porphyra yezoensis): Structure-function relationships and improved solubility. Biosci Biotechnol Biochem 59: 1933-1937, 1995.

15. Ryan JL, Glode LM and Rosenstreich DL: Lack of responsiveness of $\mathrm{C} 3 \mathrm{H} / \mathrm{HeJ}$ macrophages to lipopolysaccharide: the cellular basis of LPS-stimulated metabolism. J Immunol 122: 932-935, 1979.

16. Mosmann TR and Coffman RL: Th1 and Th2 cells: Different patterns of lymphokine secretion lead to different functional properties. Ann Rev Immunol 7: 145-173, 1989.

17. Abbas AK, Murphy KM and Sher A: Functional diversity of helper T lymphocytes. Nature 383: 787-793, 1996.

18. Kikuchi T and Sugimoto H: Detailed structure of an acidic polysaccharide in soy sauce, confirmed by use of two kinds of purified pectinases. Agric Biol Chem 40: 87-92, 1976.

19. Yang M and Cook ME: Dietary conjugated linoleic acid decreased cachexia, macrophage tumor necrosis factor- $\alpha$ production, and modifies splenocyte cytokines production. Exp Biol Med 228: $51-58,2003$

20. Sugiura H, Sugiura H, Ueya E and Ueya S: Enhanced macrophage functions and cytokine production of lymphocytes after ingestion of bon narine in female BALB/c mice. Life Sci 68: 505-515, 2000.

21. Belardelli F: Role of interferons and other cytockines in the regulation of the immune response. Acta Pathol Microbiol Immunol Scand 103: 161-179, 1995.

22. Kikuchi T and Yokotsuka T: Studies on the polysaccharides from soy sauce. Part I. Purification and properties of two acidic polysaccharides. Agric Biol Chem 36: 544-550, 1972.

23. Shida K, Makino K, Morishita A, Takamizawa K, Hachimura S, Ametani A, Sato T, Kumagai Y, Habu S and Kaminogawa S: Lactobacillus casei inhibits antigen-induced IgE secretion through regulation of cytokine production in murine splenocyte cultures. Int Arch Allergy Immunol 115: 278-287, 1998.

24. Fujiwara D, Inoue S, Wakabayashi $\mathrm{H}$ and Fujii T: The antiallergic effects of lactic acid bacteria are strain dependent and mediated by effects on both Th1/Th2 cytokine expression and balance. Int Arch Allergy Immunol 135: 205-215, 2004. 